\title{
UNIFORM CLOSURES OF FOURIER-STIELTJES ALGEBRAS
}

\author{
CHING CHOU ${ }^{1}$
}

\begin{abstract}
Let $\boldsymbol{H}$ be a closed normal subgroup of a locally compact group $G$. Assume that $f$ is a continuous function on $G$ such that it is constant on the cosets of $H$ in $G$ and it can be approximated uniformly by coefficient functions of unitary representations of $G$. We show that $f$ can be approximated uniformly by coefficient functions of representations of $G$ which are lifted from unitary representations of $G / H$. For abelian $G$, our theorem is a conjecture of R. B. Burckel.
\end{abstract}

Let $G$ be a locally compact group and $B(G)$ its Fourier-Stieltjes algebra. Then $B(G)$ is a commutative Banach algebra with its usual norm $\|\cdot\|$ (cf. Eymard [4, p. 197]). Let $C(G)$ be the algebra of bounded complex-valued continuous functions on $G$ with sup norm $\|\cdot\|_{\infty}$. The uniform closure of a set $E$ in $C(G)$ will be denoted by $E^{-}$.

For a closed normal subgroup $H$ of $G$, denote the canonical homomorphism of $G$ onto $G / H$ by $\pi$. Let $\tilde{\pi}: C(G / H) \rightarrow C(G)$ be defined by $\tilde{\pi} f=f \circ \pi, f \in C(G / H)$. The purpose of this paper is to prove the following.

THEOREM. Let $G$ be a locally compact group and $H$ a closed normal subgroup of $G$. Then

$$
\tilde{\pi}\left(B(G / H)^{-}\right)=\tilde{\pi}(C(G / H)) \cap B(G)^{-} .
$$

Recall that $B(G)$ is the algebra of coefficient functions of continuous unitary representations of $G$ or, equivalently, the algebra of linear combinations of positive definite continuous functions on $G$ (see [4]). Therefore what we stated in the Abstract is equivalent to the above theorem. If $G$ is abelian with dual group $\Gamma$ and if $M(\Gamma)$ is the Banach algebra of bounded regular Borel measures on $\Gamma$ then $B(G)=\{\hat{\mu}: \mu \in M(\Gamma)\}$ where $\hat{\mu}(x)=$ $\int_{\Gamma}(\gamma, x) d \mu(\gamma), x \in G$ and $\|\hat{\mu}\|=\|\mu\|$. In this case the above theorem can be stated as follows: Suppose $f$ is a continuous function on $G$ such that it is constant on the cosets $H+x$ and it can be approximated uniformly by Fourier-Stieltjes transforms of measures on $\Gamma$ then it can be approximated uniformly by Fourier-Stieltjes transforms of measures on the annihilator of $H$ in $\Gamma$. This is a conjecture given by Burckel in his monograph $[1, p .81$, Problem 7]. He was able to prove it if $H$ is further assumed to be compact.

Received by the editors October 12, 1978.

AMS (MOS) subject classifications (1970). Primary 43A30; Secondary 22D10, 43 A07.

Key words and phrases. Locally compact group, Fourier-Stieltjes algebra, uniform limit, invariant mean, weakly almost periodic function.

${ }^{1}$ Supported in part by NSF grant MSC 76-06377A01

(C) 1979 American Mathematical Society 
Proof of The Theorem. Eymard proved in [4, p. 202] that

$$
\tilde{\pi}(B(G / H))=\tilde{\pi}(C(G / H)) \cap B(G) .
$$

Since $\pi$ is onto, $\tilde{\pi}$ is an isometry with respect to the sup norm. Therefore, by (1), $\tilde{\pi}\left(B(G / H)^{-}\right) \subset \tilde{\pi}(C(G / H)) \cap B(G)^{-}$.

To see the converse, let $f \in \tilde{\pi}(C(G / H)) \cap B(G)^{-}$. Then, for a given $\varepsilon>0$, there exists $h \in B(G)$ such that

$$
|f(x)-h(x)|<\varepsilon, \quad x \in G .
$$

By Ryll-Nardzewski's fixed point theorem, $W(H)$, the algebra of continuous weakly almost periodic functions on $H$ with sup norm, has a unique translation invariant mean $m: m \in W(H)^{*},\|m\|=1, m \geqslant 0$ and $m(k \cdot t)=$ $m(k)$ if $k \in W(H)$ and $t \in H$ where $k \cdot t \in W(H)$ is defined by $(k \cdot t)\left(t^{\prime}\right)=$ $k\left(t^{\prime} t\right), t^{\prime} \in H$ (cf. [1, p. 15]). For $x \in G$, let $h_{x} \in C(H)$ be defined by $h_{x}(t)=h(t x), t \in H$. It is easy to check that $h_{x} \in B(H)$. Since $B(H) \subset$ $W(H)$ (cf. [1, p. 36]) $h_{1}(x)=m\left(h_{x}\right)$ is defined for each $x \in G$. We claim that

$$
h_{1} \in \tilde{\pi}(B(G / H)) \text {. }
$$

By (1), it suffices to show that (i) $h_{1} \in \tilde{\pi}(C(G / H))$ and (ii) $h_{1} \in B(G)$.

(i) Since functions in $B(G)$ are uniformly continuous, if $x_{\alpha} \rightarrow x$ in $G$ then

$$
\left|h_{1}\left(x_{\alpha}\right)-h_{1}(x)\right|=\left|m\left(h_{x_{\alpha}}-h_{x}\right)\right| \leqslant\left\|h_{x_{\alpha}}-h_{x}\right\|_{\infty} \rightarrow 0 .
$$

So $h_{1}$ is continuous on $G$. Since $m$ is $H$-invariant, $h_{1}(t x)=m\left(h_{x} \cdot t\right)=m\left(h_{x}\right)$ $=h_{1}(x)$, for $t \in H$ and $x \in G$. Therefore, $h_{1} \in \tilde{\pi}(C(G / H))$.

(ii) We will apply the following result of Davis [3, Theorem 5.1]: There exists a net of open and relatively compact subsets $U_{\alpha}$ of $H$ such that

$$
m(k)=\lim _{\alpha} \frac{1}{\lambda\left(U_{\alpha}\right)} \int_{U_{\alpha}} k(t) d \lambda(t), \quad k \in W(H)
$$

Here $\lambda$ is a fixed left Haar measure of $H$. (For locally compact amenable groups, this fact is well known; see [5, p. 43].) For each $\alpha$, set $\varphi_{\alpha}=\left(1 / \lambda\left(U_{\alpha}\right)\right)$ - $\chi_{U_{\alpha}}$ where $\chi_{U_{\alpha}}$ is the characteristic function of $U_{\alpha}$ in $H$. Let $d \mu_{\alpha}=$ $(1 / \Delta) \varphi_{\alpha}^{\sim} d \lambda$ where $\Delta$ is the modular function on $H$ and $\varphi_{\alpha}^{\sim}(t)=\varphi_{\alpha}\left(t^{-1}\right)$, $t \in H$. Then $\left\|\mu_{\alpha}\right\|=\int(1 / \Delta) \varphi_{\alpha}^{\sim} d \lambda=1$. We will consider $\mu_{\alpha}$ as a measure on $G$. Set

$$
\xi_{\alpha}(x)=\frac{1}{\lambda\left(U_{\alpha}\right)} \int_{U_{\alpha}} h(t x) d \lambda(t), \quad x \in G .
$$

Then $\xi_{\alpha}=\mu_{\alpha} * h$. By [4, p. 198], $\xi_{\alpha} \in B(G)$ and $\left\|\xi_{\alpha}\right\|<\left\|\mu_{\alpha}\right\|\|h\|=\|h\|$. By (4), $h_{1}(x)=m\left(h_{x}\right)=\lim _{\alpha} \xi_{\alpha}(x), x \in G$. Therefore, $h_{1}$ is a pointwise limit of a net of functions $\xi_{\alpha}$ in $B(G)$ with $\left\|\xi_{\alpha}\right\|<\|h\|$. Since $h_{1}$ is continuous, by [4, p. 202], $h_{1} \in B(G)$. The proof of (ii) is completed.

Note that for each $t \in H, f_{x}(t)=f(x t)=f(x)$. Therefore, $m\left(f_{x}\right)=f(x)$. Now, by (2), $\left\|f_{x}-h_{x}\right\|_{\infty}<\varepsilon$ and hence $\left|m\left(f_{x}\right)-m\left(h_{x}\right)\right|<\varepsilon, x \in G$. So, we 
have

$$
\left|f(x)-h_{1}(x)\right|<\varepsilon, \quad x \in G .
$$

By assumption there exists $g \in C(G / H)$ such that $\tilde{\pi} g=f$ and by (3) there exists $h_{2} \in B(G / H)$ such that $\tilde{\pi}\left(h_{2}\right)=h_{1}$. So (5) can be written as

$$
\left|g(H x)-h_{2}(H x)\right|<\varepsilon, \quad H x \in G / H .
$$

Since $\varepsilon>0$ is arbitrary, we conclude that $g \in B(G / H)^{-}$and hence $f=\tilde{\pi}(g)$ $\in \tilde{\pi}\left(B(G / H)^{-}\right)$.

REMARK. If, in the above theorem, $H$ is compact, then the fact that $h_{1} \in B(G)$, the crux of our proof, becomes obvious. For, in this case, $h_{1}=\lambda * h$ where $\lambda$ is the normalized Haar measure of $H$. If $G$ is abelian, $H$ is compact, i.e., the case considered by Burckel [1, Theorem A.39], and $h=\hat{\mu} \in$ $B(G)$ then $h_{1}=\hat{\mu}_{1}$ where $\mu_{1}$ is defined by $\mu_{1}(B)=\mu(B \cap \Lambda), B$ a Borel set in $\Gamma$ and $\Lambda$ the annihilator of $H$ in $\Gamma$ :

$$
\begin{aligned}
h_{1}(x) & =\int_{H} h(x+t) d \lambda(t) \\
& =\int_{\Gamma}(\gamma, x)\left(\int_{H}(\gamma, t) d \lambda(t)\right) d \mu(\gamma) \\
& =\int_{\Lambda}(\gamma, x) d \mu(\gamma)=\hat{\mu}_{1}(x), \quad x \in G .
\end{aligned}
$$

Note that Burckel proved his Theorem A.39 by applying Ramirez' characterization theorem for $B(G)^{-}$(cf. [1, Theorem A.38]). He then applied his Theorem A.39 to give a proof of the following theorem of Ramirez [6]: If $G$ is a noncompact locally compact abelian group then $B(G)^{-} \varsubsetneqq W(G)$ (cf. [1, p. 67]).

Let $G$ and $H$ be as in our theorem. It is known that $\tilde{\pi}(W(G / H))=$ $\tilde{\pi}(C(G / H)) \cap W(G)$ (see [1]). Therefore we have the following.

Corollary 1. If $B(G)^{-}=W(G)$ then $B(G / H)^{-}=W(G / H)$.

By combining this corollary with Ramirez' theorem in [6] we can state the following.

COROLlary 2. Suppose $G$ is a locally compact group with a noncompact locally compact abelian quotient group. Then $W(G) \supsetneqq B(G)^{-}$.

For example, $W(G) \supsetneqq B(G)^{-}$if $G$ is the Heisenberg group or the $a x+b$ group. Note that there exist noncompact groups $G$ with $W(G)=B(G)^{-}$, see [2].

\section{REFERENCES}

1. R. B. Burckel, Weakly almost periodic functions on semigroups, Gordon and Breach, New York, 1970. 
2. C. Chou, Minimally weakly almost periodic groups, J. Functional Anal. (to appear).

3. H. W. Davis, Generalized almost periodicity in groups, Trans. Amer. Math. Soc. 191 (1974), 329-352.

4. P. Eymard, L'algèbre de Fourier d'un groupe localement compact, Bull. Soc. Math. France 92 (1964), 181-236.

5. F. P. Greenleaf, Invariant means on topological groups and their applications, Van NostrandReinhold, New York, 1969.

6. D. Ramirez, Weakly almost periodic functions and Fourier-Stieltjes transforms, Proc. Amer. Math. Soc. 19 (1968), 1087-1088.

Department of Mathematics, State University of New York at Buffalo, Buffalo, New YoRK 14214 